\title{
Ultradiscretization of reaction-diffusion type partial differential equations exhibiting pulse propagation
}

\author{
Y. Yamazaki* and S. Ohmori \\ Department of Physics, Department of Physics, School of Advanced Science and Engineering, \\ Waseda University, Shinjuku, Tokyo 169-8555, Japan
}

*yoshy@waseda.jp

PACS 05.90. $+\mathrm{m}, 02.90 .+p$

DOI 10.17586/2220-8054-2017-8-1-38-41

Cellular automaton rules are derived from a set of reaction-diffusion partial differential equations by means of ultradiscretization method. The rules reproduce dynamical properties of pulse: annihilation, soliton-like preservation and periodic generation with a pacemaker.

Keywords: ultradiscretization, tropical discretization, pulse dynamics, cellular automaton, reaction-diffusion systems.

Received: 20 July 2016

Revised: 30 July 2016

\section{Introduction: ultradiscretization}

Now we carry out ultradiscretization of the following reaction-diffusion system [1,2]:

$$
\left\{\begin{aligned}
\tau_{u} \frac{\partial u}{\partial t} & =D_{u} \frac{\partial^{2} u}{\partial x^{2}}-(u-\alpha)(u-\beta)(u-\gamma)-v \\
\tau_{v} \frac{\partial v}{\partial t} & =D_{v} \frac{\partial^{2} v}{\partial x^{2}}+\kappa u-\lambda v+i
\end{aligned}\right.
$$

where $\tau_{u}, \tau_{v}, D_{u}, D_{v}, \kappa, \lambda$, and $i$ are positive constants. $\alpha, \beta$, and $\gamma$ are also positive and satisfy $0<\alpha<\beta<\gamma$.

For Eq. (1), we first adopt the following tropical discretization [3,4]:

$$
u_{j}^{n+1}=w_{j}^{n} \frac{w_{j}^{n}+F\left(w_{j}^{n}\right) \Delta t}{w_{j}^{n}+G\left(w_{j}^{n}, v_{j}^{n}\right) \Delta t},
$$

where $w_{j}^{n}=\mu\left(u_{j+1}^{n}+u_{j-1}^{n}\right)+(1-2 \mu) u_{j}^{n}$ and $\mu=\frac{D_{u} \Delta t}{\tau_{u}(\Delta x)^{2}} . \quad F$ and $G$ are defined as $F(u)=\frac{1}{\tau_{u}}\left[(\alpha+\beta+\gamma) u^{2}+\alpha \beta \gamma\right]$ and $G(u, v)=\frac{1}{\tau_{u}}\left[u^{3}+(\alpha \gamma+\alpha \beta+\beta \gamma) u+v\right]$, respectively. For Eq. (3), the ultradiscrete limits [5-8]:

$$
\begin{cases}\lim _{\varepsilon \rightarrow+0} \varepsilon \log \left(e^{A / \varepsilon}+e^{B / \varepsilon}+\cdots\right) & =\max (A, B, \ldots), \\ \lim _{\varepsilon \rightarrow+0} \varepsilon \log \left(e^{A / \varepsilon} \cdot e^{B / \varepsilon} \cdots\right) & =A+B+\cdots\end{cases}
$$

are carried out after the following variable transformations are performed:

$$
\left\{\begin{array}{l}
\Delta t / \tau_{u}=e^{T / \varepsilon} /\left(\mu^{n+1}\right)^{2}, \quad u_{j}^{n}=\mu^{n} e^{U_{j}^{n} / \varepsilon}, \quad v_{j}^{n}=\left(\mu^{n+1}\right)^{3} e^{V_{j}^{n} / \varepsilon}, \\
\alpha=\mu^{n+1} e^{A / \varepsilon}, \quad \beta=\mu^{n+1} e^{B / \varepsilon}, \quad \gamma=\mu^{n+1} e^{\Gamma / \varepsilon}, \\
(1-2 \mu) / \mu=e^{M / \varepsilon} .
\end{array}\right.
$$

Then, the ultradiscrete equation is obtained by considering $A<B<\Gamma$ as:

$$
\begin{aligned}
U_{j}^{n+1}= & W_{j}^{n}+\max \left\{W_{j}^{n}, T+\max \left(\Gamma+2 W_{j}^{n}, A+B+\Gamma\right)\right\} \\
& -\max \left\{W_{j}^{n}, T+\max \left(3 W_{j}^{n}, B+\Gamma+W_{j}^{n}, V_{j}^{n}\right)\right\},
\end{aligned}
$$

where $W_{j}^{n}=\max \left(U_{j+1}^{n}, M+U_{j}^{n}, U_{j-1}^{n}\right)$.

Next, the discretized equation of Eq. (2):

$$
\tau_{v} \frac{v_{j}^{n+1}-v_{j}^{n}}{\Delta t}=D_{v} \frac{v_{j+1}^{n}-2 v_{j}^{n}+v_{j-1}^{n}}{(\Delta x)^{2}}+\kappa u_{j}^{n}-\lambda v_{j}^{n}+i
$$


can be rewritten as follows for ultradiscretization:

$$
v_{j}^{n+1}=\theta\left(v_{j+1}^{n}+v_{j-1}^{n}\right)+\left(1-2 \theta-\frac{\lambda \Delta t}{\tau_{v}}\right) v_{j}^{n}+\frac{\Delta t \kappa}{\tau_{v}} u_{j}^{n}+\frac{\Delta t i}{\tau_{v}},
$$

where $\theta=\left(D_{v} \Delta t\right) /\left((\Delta x)^{2} \tau_{v}\right)$. Applying the variable transformations

$$
v_{j}^{n}=\theta^{n} e^{V_{j}^{n} / \varepsilon}, \quad \frac{1}{\theta}\left(1-2 \theta-\frac{\lambda \Delta t}{\tau_{v}}\right)=e^{N / \varepsilon}, \quad \frac{\Delta t \kappa}{\tau_{v}}=\frac{\theta^{n+1} e^{S / \varepsilon}}{\mu^{n}}, \quad \frac{\Delta t i}{\tau_{v}}=\theta^{n+1} e^{I / \varepsilon},
$$

where $\left(\mu^{n+1}\right)^{3}=\theta^{n}$, we obtain the following ultradiscrete equation through Eq. (4):

$$
V_{j}^{n+1}=\max \left(L_{j}^{n}, S+U_{j}^{n}, I\right) .
$$

Here, $L_{j}^{n}=\max \left(V_{j+1}^{n}, N+V_{j}^{n}, V_{j-1}^{n}\right)$. Then, we obtain the set of ultradiscrete equations (6) and (10) from Eqs. (1) and (2).

The transition from $W_{j}^{n}$ to $U_{j}^{n+1}$ using Eq. (6) is shown in Fig. 1 as a function of $W_{j}^{n}$ and $V_{j}^{n}$. Fig. 1 shows that the convergence of $U_{j}^{n}$ to $A$ or $\Gamma$ depends on the value of $V_{j}^{n}$. There is a time $n$ at which the state $U_{j}^{n}$ becomes $A$ or $\Gamma$ in regions I and IV. This fact reflects the ultradiscrete bistability in Eq. (6). Here, ultradiscrete bistability means that for the initial value $U_{j}^{0}$, which is different from the two stable points, there exists a finite time $n$ such that the state $U_{j}^{n}$ becomes one of the two stable points. Note also that $U_{j}^{n+1}=A$ when $V_{j}^{n}=3 W_{j}^{n}+\Gamma-A$ for $W_{j}^{n}>(A+B) / 2$ and $U_{j}^{n+1}=\Gamma$ when $V_{j}^{n}=3 W_{j}^{n}$ for $W_{j}^{n}>(B+\Gamma) / 2$.

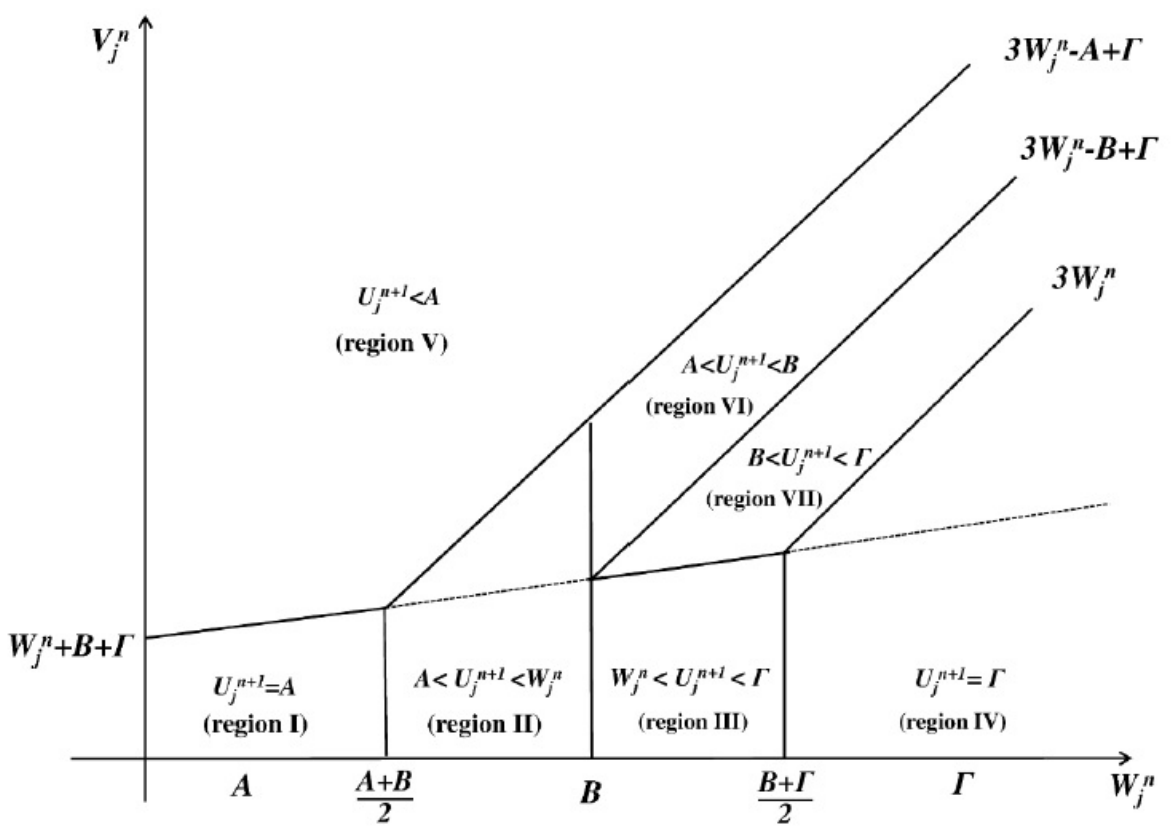

FIG. 1. Transition diagram of $U_{j}^{n}$ from $n$ to $n+1$. The regions (I - VII) are separated by solid lines

\section{Cellular automaton rules}

In order to derive two-state cellular automaton $(\mathrm{CA})$ rules from the above set of ultradiscrete equations (6) and (10), we assume the following additional relation:

$$
\text { If } W_{j}^{n}=A \text {, then } U_{j}^{n+1} \equiv A \text {. }
$$

Now we consider the conditions $M=0$ or $M \leq A-\Gamma, T \geq \max \{0,-(A+B+\Gamma)\}, N=0, S=3 \Gamma-A$, and $I<3 \Gamma$. Under these conditions, $W_{j}^{n}, U_{j}^{n} \in\{A, \Gamma\}$ is confirmed.

When $V_{j \pm 1}^{n}, V_{j}^{n} \leq 3 \Gamma-A+U_{j}^{n}$, we obtain from Eq. (10)

$$
V_{j}^{n+1}=3 \Gamma-A+U_{j}^{n} .
$$

From $W_{j}^{n} \in\{A, \Gamma\}$ and $T \geq \max \{0,-(A+B+\Gamma)\}$, Eq. (6) can be rewritten as

$$
U_{j}^{n+1}=W_{j}^{n}+\max \left(T+\Gamma+2 W_{j}^{n}, T+A+B+\Gamma\right)-\max \left(T+3 W_{j}^{n}, T+B+\Gamma+W_{j}^{n}, T+V_{j}^{n}\right)
$$


using the relation $\Gamma<T+3 \Gamma$. Considering Eq. (12), this equation is described as the following function of $U_{j}^{n}$, $U_{j \pm 1}^{n}$, and $U_{j}^{n-1}$ :

$$
U_{j}^{n+1}=W_{j}^{n}+\max \left(\Gamma+2 W_{j}^{n}, A+B+\Gamma\right)-\max \left(3 W_{j}^{n}, B+\Gamma+W_{j}^{n}, 3 \Gamma-A+U_{j}^{n-1}\right),
$$

where $W_{j}^{n}=\max \left(U_{j+1}^{n}, U_{j}^{n}+M, U_{j-1}^{n}\right)$. Taking Eq. (11) into account, we obtain the following CA rules.

$$
\text { If } W_{j}^{n}=\left\{\begin{array}{ll}
A, & \text { Eq.(11) } \\
\Gamma, & \text { Eq.(13) }
\end{array}\right\} \text { is adopted. }
$$

Note that Eq. (14) produces the following two CA rules depending on the conditions of $M$.

When $M=0$, the rule shown in Table 1 is obtained from Eq. (14). The values of $\left\{U_{j}^{0}\right\}$ and $\left\{U_{j}^{1}\right\}$ have to be given as the initial conditions. Fig. 2 shows the spatiotemporal patterns obtained from Table 1 for two different initial conditions. Black and white cells correspond to $\Gamma$ and $A$, respectively. The initial conditions are set as follows. (a) For $j$ and $k(>j), U_{j}^{0}=U_{j}^{1}=\Gamma$ and $U_{k}^{0}=U_{k}^{1}=\Gamma$. Otherwise, $U^{0}=U^{1}=A$. (b) Using the same $j$ and $k$ as in (a), $U_{j}^{0}=U_{j}^{1}=\Gamma$ and $U_{k+1}^{0}=U_{k+1}^{1}=\Gamma$. Otherwise, $U^{0}=U^{1}=A$. It is found that the two pulse-like cells with $U=\Gamma$ are created periodically from the initial cell with $U=\Gamma$ and that the two pulse-like cells propagate in both directions. The difference between Figs. 2(a) and 2(b) is the initial relative positions between the two pulse-like cells. Regardless of such a difference in the initial conditions, both Figs. 2(a) and 2(b) show that the two pulses are annihilated when they collide with each other.

TABLE 1. CA rule given by Eq. (14) with $M=0$. A value of $U_{j}^{n+1}$ in parentheses is calculated using the value of $U_{j}^{n-1}$ in parentheses

\begin{tabular}{|c|c|c|c|c|c|c|c|c|}
\hline$U_{j}^{n-1}$ & $\Gamma(A)$ & $\Gamma(A)$ & $\Gamma(A)$ & $\Gamma(A)$ & $\Gamma(A)$ & $\Gamma(A)$ & $\Gamma(A)$ & $\Gamma(A)$ \\
\hline$U_{j+1}^{n}, U_{j}^{n}, U_{j-1}^{n}$ & $\Gamma, \Gamma, \Gamma$ & $\Gamma, \Gamma, A$ & $\Gamma, A, \Gamma$ & $A, \Gamma, \Gamma$ & $\Gamma, A, A$ & $A, \Gamma, A$ & $A, A, \Gamma$ & $A, A, A$ \\
\hline$W_{j}^{n}$ & $\Gamma$ & $\Gamma$ & $\Gamma$ & $\Gamma$ & $\Gamma$ & $\Gamma$ & $\Gamma$ & $A$ \\
\hline$U_{j}^{n+1}$ & $A(\Gamma)$ & $A(\Gamma)$ & $A(\Gamma)$ & $A(\Gamma)$ & $A(\Gamma)$ & $A(\Gamma)$ & $A(\Gamma)$ & $A(A)$ \\
\hline
\end{tabular}

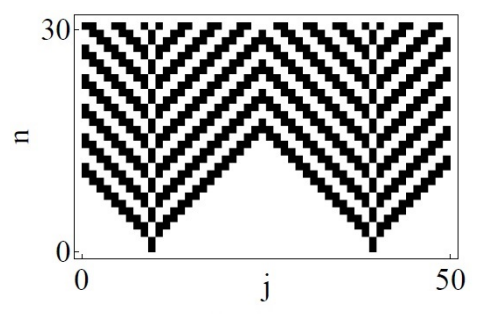

(a)

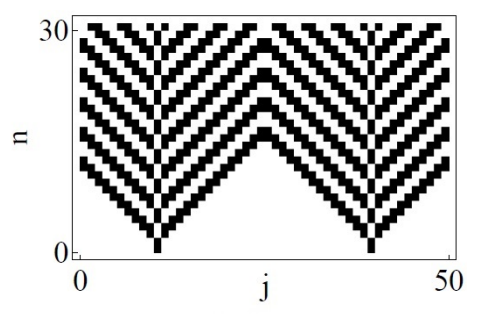

(b)

FIG. 2. Spatiotemporal patterns obtained according to Table 1 from two cells with $U=\Gamma$ (50 cells and 30 time steps). The difference between (a) and (b) is the initial relative position between the two cells with $U=\Gamma$. Time steps proceed from bottom to top. Black and white cells correspond to $\Gamma$ and $A$, respectively

Alternatively, when $M \leq A-\Gamma, W_{j}^{n}=\max \left(U_{j+1}^{n}, U_{j-1}^{n}\right)$, the rule shown in Table 2 is obtained from Eq. (14). Fig. 3 shows spatiotemporal patterns obtained from Table 2 for two different initial conditions. Black and white cells correspond to $\Gamma$ and $A$, respectively. As the initial conditions, we set the followings: (a) For $j$ and $k(>j+2), U_{j}^{0}=U_{j+1}^{1}=\Gamma$ and $U_{k}^{0}=U_{k-1}^{1}=\Gamma$. Otherwise, $U^{0}=U^{1}=A$. (b) Using the same $j$ and $k$ as in (a), $U_{j}^{0}=U_{j+1}^{1}=\Gamma$ and $U_{k+1}^{0}=U_{k}^{1}=\Gamma$. Otherwise, $U^{0}=U^{1}=A$. Figs. 3(a) and 3(b) show that there are two types of collision of propagating cells with $U=\Gamma$, namely, soliton-like preservation and annihilation, respectively. The emergence of these two different types of collision depends on the relative positions between the two pulse-like cells. If a pulse-like cell at $U_{j}^{n}$ encounters another one at $U_{j+1}^{n}$, they pass through each other, and the pulses are preserved. However, if the two pulses exist at the same position at the same time, they annihilate. 
TABLE 2. CA rule given by Eq. (14) with $M \leq A-\Gamma$. A value of $U_{j}^{n+1}$ in parentheses is calculated using the value of $U_{j}^{n-1}$ in parentheses

\begin{tabular}{|c|c|c|c|c|c|c|c|c|}
\hline$U_{j}^{n-1}$ & $\Gamma(A)$ & $\Gamma(A)$ & $\Gamma(A)$ & $\Gamma(A)$ & $\Gamma(A)$ & $\Gamma(A)$ & $\Gamma(A)$ & $\Gamma(A)$ \\
\hline$U_{j+1}^{n}, U_{j}^{n}, U_{j-1}^{n}$ & $\Gamma, \Gamma, \Gamma$ & $\Gamma, \Gamma, A$ & $\Gamma, A, \Gamma$ & $A, \Gamma, \Gamma$ & $\Gamma, A, A$ & $A, \Gamma, A$ & $A, A, \Gamma$ & $A, A, A$ \\
\hline$W_{j}^{n}$ & $\Gamma$ & $\Gamma$ & $\Gamma$ & $\Gamma$ & $\Gamma$ & $A$ & $\Gamma$ & $A$ \\
\hline$U_{j}^{n+1}$ & $A(\Gamma)$ & $A(\Gamma)$ & $A(\Gamma)$ & $A(\Gamma)$ & $A(\Gamma)$ & $A(A)$ & $A(\Gamma)$ & $A(A)$ \\
\hline
\end{tabular}

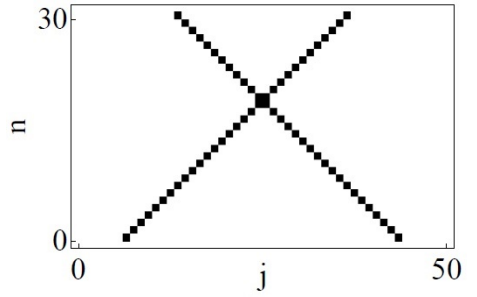

(a)

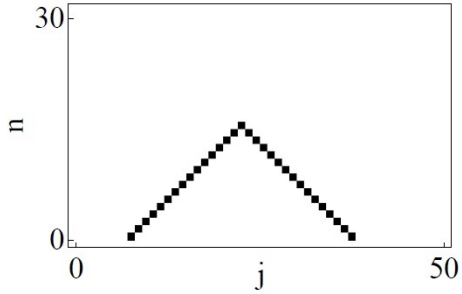

(b)

FIG. 3. Spatiotemporal patterns obtained from Table II. (a) soliton-like preservation and (b) pulse annihilation (50 cells and 30 time steps). Time steps proceed from bottom to top. Black and white cells correspond to $\Gamma$ and $A$, respectively

\section{Conclusion}

We have derived the ultradiscretized equations [Eqs. (6) and (10)] from the reaction-diffusion system Eqs. (1) and (2). By considering the $V$ dependence on the state transition of $U$, we have obtained the CA rules from the ultradiscretized equations. It was shown that some $\mathrm{CA}$ rules reproduce the spatiotemporal properties of pulse dynamics: pulse annihilation, periodic pulse generation from a pacemaker and soliton-like preservation. (More details are shown in ref. [9].)

\section{Acknowledgements}

The authors are grateful to Professor Daisuke Takahashi, Professor Tomoyuki Yamamoto, and Emeritus Professor Akihiko Kitada for useful discussions and encouragement.

\section{References}

[1] Ohta T., Hayase Y., and Kobayashi R. Spontaneous formation of concentric waves in a two-component reaction-diffusion system. Phys. Rev. E., 1996, 54, P. 6074.

[2] Hayase Y. Collision and Self-Replication of Pulses in a Reaction Diffusion System. J. Phys. Soc. Jpn., 1997, 66, P. 2584.

[3] Murata M. Tropical discretization: ultradiscrete FisherKPP equation and ultradiscrete AllenCahn equation. J. Differ. Equations Appl., 2013, 19, P. 1008.

[4] Murata M. Multidimensional traveling waves in the AllenCahn cellular automaton. J. Phys. A., 2015, 48, P. 255202.

[5] Tokihiro T., Takahashi D., Matsukidaira J. and Satsuma J. From Soliton Equations to Integrable Cellular Automata through a Limiting Procedure. Phys. Rev. Lett., 1996, 76, P. 3247.

[6] Grammaticos B., Ohta Y., Ramani A., Takahashi D. and Tamizhmani K.M. Cellular automata and ultra-discrete Painlevé equations. Phys. Lett. A., 1997, 226, P. 53.

[7] Matsuya K. and Murata M. Spatial pattern of discrete and ultradiscrete Gray-Scott model. Discrete Contin. Dyn. Syst. Ser. B., 2015, 20, P. 173.

[8] Takahashi D., Shida A. and Usami M. On the pattern formation mechanism of (2+1)D max-plus models. J. Phys. A., 2001,34, P. 10715.

[9] Ohmori S., Yamazaki Y. Cellular Automata for Spatiotemporal Pattern Formation from ReactionDiffusion Partial Differential Equations. $J$. Phys. Soc. Jpn., 2016, 85, P. 014003. 